\title{
Power-Scalable, Sub-Nanosecond Mode-Locked Erbium-Doped Fiber Laser Based on a Frequency-Shifted-Feedback Ring Cavity Incorporating a Narrow Bandpass Filter
}

\author{
Luis Alonso Vazquez-Zuniga and Yoonchan Jeong* \\ Laser Engineering and Applications Laboratory, Department of Electrical and Computer Engineering, \\ Seoul National University, 599 Gwanak-ro, Gwanak-gu, Seoul 151-744, Korea
}

(Received March 8, 2013 : revised March 18, 2013 : accepted March 18, 2013)

\begin{abstract}
We present an all-fiberized power-scalable, sub-nanosecond mode-locked laser based on a frequencyshifted-feedback ring cavity comprised of an erbium-doped fiber, a downshifting acousto-optic modulator (AOM), and a bandpass filter (BPF). With the aid of the frequency-shifted feedback mechanism provided by the AOM and the narrow filter bandwidth of $0.45 \mathrm{~nm}$, we generate self-starting, mode-locked optical pulses with a spectral bandwidth of $\sim 0.098 \mathrm{~nm}$ and a pulsewidth of 432 to $536 \mathrm{ps}$. In particular, the output power is readily scalable with pump power while keeping the temporal shape and spectral bandwidth. This is obtained via the consolidation of bound pulse modes circulating at the fundamental repetition rate of the cavity. In fact, the consolidated pulses form a single-entity envelope of asymmetric Gaussian shape where no discrete internal pulses are perceived. This result highlights that the inclusion of the narrow $\mathrm{BPF}$ into the cavity is crucial to achieving the consolidated pulses.
\end{abstract}

Keywords: Mode-locked lasers, Erbium fiber lasers, Frequency-shifted feedback lasers

OCIS codes : (140.3510) Lasers, fiber; (140.3500) Lasers, erbium; (140.4050) Mode-locked lasers; (320.5550) Pulses

\section{INTRODUCTION}

The lasing mechanism of a frequency-shifted feedback (FSF) laser (FSFL) is somewhat different from that of a conventional laser because it is formed by successive frequency shifts of the spectral components of the cavity modes [1]. This means that in terms of the spectral cavity mode, it cannot have a steady-state condition. As a result, one can obtain a variety of unique and distinctive lasing properties from these types of lasers. Therefore, FSFLs have been investigated as coherent light sources for various applications, such as broadband continuous-wave $(\mathrm{CW})$ lasers [1, 2], multiwavelength lasers [3-5], and pulsed lasers [6-12]. In general, the FSF mechanism is obtained by an acousto-optic modulator (AOM), either an upshifting or downshifting type, inserted in the laser cavity. (It should be noted that the function of the AOM in the cavity is not for the amplitude modulation of the cavity modes but for their continuous frequencyshift.)

In particular, stable mode-locked pulses can also be generated from FSFLs, and pulse durations obtained with them are typically in the order of tens of picoseconds [6-9]. To date, most experimental investigations of FSFLs have focused on achieving shorter pulses through modifying cavity configurations [10-12], or by combining the FSF mechanism with other mode-locking techniques, e.g., nonlinear polarization rotation $[10,12]$. Such novel configurations were able to reduce pulse durations to a few picoseconds or hundreds of femtoseconds. An interesting feature commonly observed in FSFLs is that their pulse durations can also be extended to a few hundred picoseconds or sub-nanoseconds via the formation of bound multiple-trailing pulses [9], thereby being capable of pulse-energy scaling. However, in such sub-nanosecond regimes, the bound multiple-trailing pulses tend to have non-negligible splits among the internal pulses, giving rise to strong internal modulation across the whole group of pulses [9]. It should be noted that the mechanism of the bound multiple-trailing pulses in FSFL regimes is far different from that of bunching of random pulses in noise-like pulse regimes $[13,14]$. Here, we investigate an all-fiberized FSFL

\footnotetext{
*Corresponding author: yoonchan@snu.ac.kr

Color versions of one or more of the figures in this paper are available online.
} 
that can generate sub-nanosecond mode-locked pulses having no internal pulse-separations by means of incorporating a bandpass filter (BPF) of a narrow bandwidth. As a result, the bound pulses form a single-entity envelope of an asymmetric Gaussian shape, which is in agreement with the theoretical prediction previously reported [13], while we consider that the cavity dispersion also plays an important role in forming the asymmetry. We found that this type of pulse can also offer good power scalability via broadening the pulsewidth while increasing pump power. In the following section we discuss its detailed experimental demonstration and analysis.

\section{EXPERIMENTAL RESULT}

The schematic of the all-fiberized FSFL is shown in Fig. 1. It consists of 2-m of an erbium-doped fiber (EDF, Fibercore) that has a small-signal absorption rate of 37 $\mathrm{dB} / \mathrm{m}$ at $1530 \mathrm{~nm}$. It is pumped by two laser diodes (LDs) at $1480 \mathrm{~nm}$ through a wavelength-division-multiplexed (WDM) coupler $(1480 / 1550 \mathrm{~nm})$. A second WDM coupler spliced at the other end of the EDF removes the unabsorbed pump light from the cavity. The combination of two sets of polarization controllers (PCs, $\mathrm{PC} 1$ and $\mathrm{PC} 2$ ) and a fiberized polarization beam splitter (PBS) are utilized to introduce the phase bias required to lock the longitudinal modes of the ring cavity and to adjust the output coupling ratio (OCR) of the cavity. PC1 and PC2 consist of three wave plates $(\lambda / 4-\lambda / 2-\lambda / 4)$ and two wave plates $(\lambda / 4-\lambda / 2)$, respectively. A tap coupler (95:5) after PC2 is used to monitor the intra-cavity signal. The frequency-shifting mechanism is obtained through an AOM located inside the cavity. For this, we investigate the use of two different fiber-coupled AOMs downshifting at $110 \mathrm{MHz}$ and 200 $\mathrm{MHz}$ (Gooch \& Housego). (The AOM frequencies were chosen due mainly to their availability at the time of the experiment. In general, the amount of frequency-shift generated by the AOM is related to the spectral bandwidth of the resultant laser signal [6]. That is, the longer frequency-shift will give rise to the broader spectral bandwidth. However, it does not critically affect the stability of the mode-locking [6].) Their insertion losses are 3 and $7 \mathrm{~dB}$ for the 110-

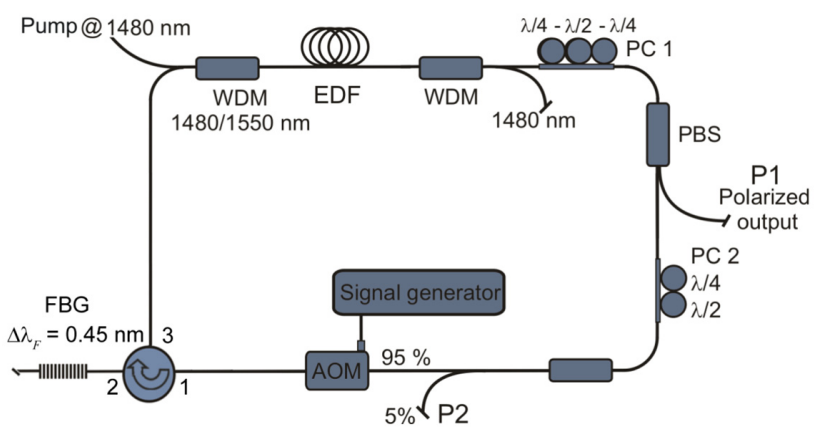

FIG. 1. Schematic of the all-fiberized FSFL. and $200-\mathrm{MHz}$ AOMs, respectively. The AOMs are driven by a radio-frequency (RF) signal generator. In addition, the cavity incorporates a BPF, which is based on a uniform fiber Bragg grating (FBG) with a spectral bandwidth $\left(\Delta \lambda_{F}\right)$ of $0.45-\mathrm{nm}$ measured at full width at half maximum (FWHM) and reflectivity (R) of $99 \%$ combined with a fiberized 3 -port circulator whose insertion loss is $\sim 3 \mathrm{~dB}$. It should be noted that since the FBG is based on an unchirped, uniform one, the group-delay change due to the dispersion of the FBG is negligible. The unidirectional operation of the ring cavity is ensured via the circulator which has an isolation of $\sim 40 \mathrm{~dB}$ at $\sim 1550 \mathrm{~nm}$. The total cavity length becomes $\sim 23.5 \mathrm{~m}$ and has an average group velocity dispersion (GVD) parameter of $\beta_{2}=-0.395 \mathrm{ps}^{2}$ at $\sim 1550 \mathrm{~nm}$, which implies that the whole cavity is in an anomalous regime. The optical spectrum is measured with an optical spectrum analyzer (OSA), while the time trace of the signal is measured with the optical channel of a digital communication analyzer (DCA) having a 22-GHz bandwidth.

When the cavity shown in Fig. 1 was initially tested without incorporating the AOM, it was completely impossible to make the laser mode-locked at any pump power level up to $250 \mathrm{~mW}$ in the given condition. This indicates that the saturable absorber effect induced by the nonlinear polarization evolution combined with the PBS in the cavity was not strong enough to generate mode-locked pulses, thereby only forming noisy, random pulses in the time domain. On the contrary, when the 110-MHz AOM was incorporated in the cavity, mode-locked pulses were readily achieved. It is worth mentioning that the laser was set to operate in the non-resonant regime since mode-locking was achieved more easily and kept with higher stability. This means that the driving frequency of the AOM was detuned from a harmonic of the fundamental cavity resonance frequency [9].

Figure 2 shows a typical output pulse formation when

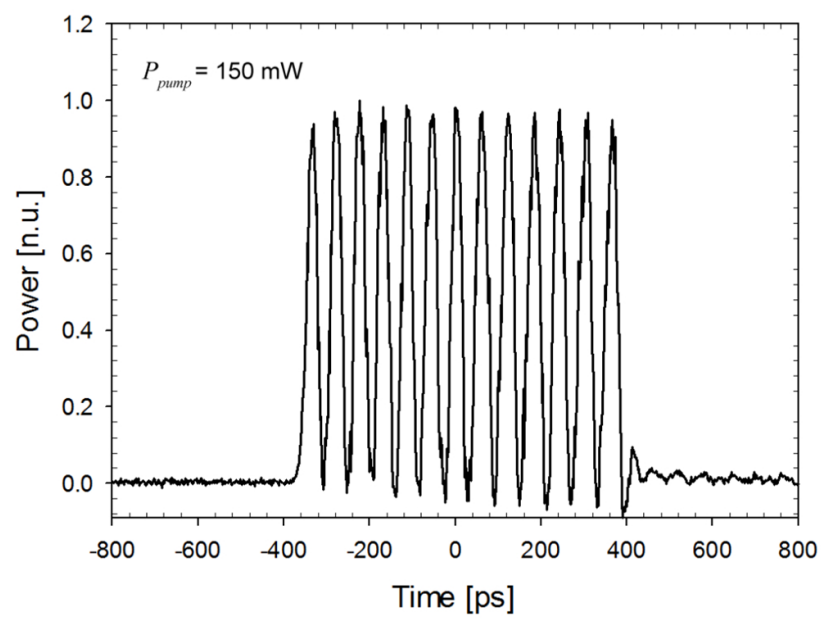

FIG. 2. Time trace of the output signal of the FSFL comprising a $110-\mathrm{MHz}$ downshifting $\mathrm{AOM}$ and an in-line BPF with $\Delta \lambda_{F}=1.3 \mathrm{~nm}$. (n.u.: normalized unit). 
the laser was operating in a multiple-pulse mode-locked (MPML) regime where an in-line BPF having a filter bandwidth of $1.3 \mathrm{~nm}$, instead of $0.45 \mathrm{~nm}$, was utilized in the cavity similar to the one shown in Fig. 1. In this regime, the laser presented self-starting mode-locked behaviors for pump powers in excess of $150 \mathrm{~mW}$, and the number of the bound consecutive pulses increased with pump power. However, as can be seen in Fig. 2, the consecutive pulses could not form a single-entity envelop. In fact, the formation of these multiple pulses resembles the soliton-mode-locked regimes operating with excessive gain [9]. Since the net dispersion of the cavity is in the anomalous regime, we attribute the formation of the bound consecutive pulses to quasi-soliton formation in the cavity section where dispersion is anomalous. In this case the self-phase modulation (SPM) plays an important role in forming the pulse, and, thus, we expect that the peak power and pulse shape basically follow the soliton-area theorem [15]. Consequently, consecutive quasisoliton pulses cannot join together because of the strong soliton interaction between them $[13,16]$, thereby resulting in a clear pulse separation between them. Therefore, in order for the FSFL to operate in a consolidated-pulse mode-locked (CPML) regime having no internal pulse separations, the SPM effects must be substantially alleviated unless the dispersion characteristic of the cavity is altered. For this purpose, we reduce the filter bandwidth, which can subsequently decrease the instantaneous intensity of the resultant pulse via hindering the formation of short pulses in the cavity. Here, we utilized the FBG with spectral bandwidth of 0.45 $\mathrm{nm}$ (FWHM) in the cavity, as described earlier in this section.

With incorporating the FBG-based BPF, we observed self-starting, fundamental mode-locked pulses $\left(f_{0}=8.51 \mathrm{MHz}\right)$ for pump powers in excess of $200 \mathrm{~mW}$. The increase of the lasing threshold in comparison with the case with the 1.3-nm BPF, was due mainly to the additional insertion loss $(\sim 3 \mathrm{~dB})$ incurred by the 3-port circulator combined with the FBG. Fig. 3 shows the pulse spectrum and time trace obtained from the FSFL incorporating the BPF. One can clearly see that it forms a consolidated pulse with a single-entity envelope having no internal pulse separations. As the minimum pulsewidth resolvable by the DCA is $\sim 22$ ps, one may argue that our measurement is resolution-limited. However, based on the spectral bandwidth $(\Delta \lambda)$ of 0.098 $\mathrm{nm}$ (FWHM) generated by the FSFL operating in the current CPML regime, the transform-limited pulse can only be as short as a pulse duration of $\sim 25$ ps (assuming a sech $^{2}$ pulse shape). This is long enough to be resolved by the DCA. In addition, one can notice that the output spectrum is asymmetric, which is due mainly to the asymmetric gain spectrum across the spectral range close to the filter edge via the continuous downshifting of the cavity spectral components [6]. That is, under a downshifting FSF regime, blue-shifted components invariably see higher gain than redshifted components across the spectral range close to the filter edge, so that the spectral peak must be in the shorter- wavelength side as seen in Fig. 3(a). This can further explain the asymmetry of the temporal pulse shape shown in Fig. 3(b), where we can see the boosted, slow-increasing leading edge and the suppressed, fast-decreasing trailing edge since the blue-shifted components travel in the leading edge and the red-shifted components in the trailing edge in the anomalous regime for the case where the SPM is negligible.

In addition, we could see that the pulsewidth of the consolidated pulse as well as its pulse-energy was scaled with pump power. The pulsewidth and average output power as a function of the pump power are shown in Fig. 4, which indicate a good linearity with respect to the pump power. The pulse-energy is readily obtained via dividing the average output power by the repetition rate of 8.51 $\mathrm{MHz}$ as the background amplified spontaneous emission (ASE) was negligible. Since the maximum output power was limited by the maximum pump power used, which was $\sim 250 \mathrm{~mW}$, we expect that further power scaling will be possible if higher pump power is launched in the cavity. It is also

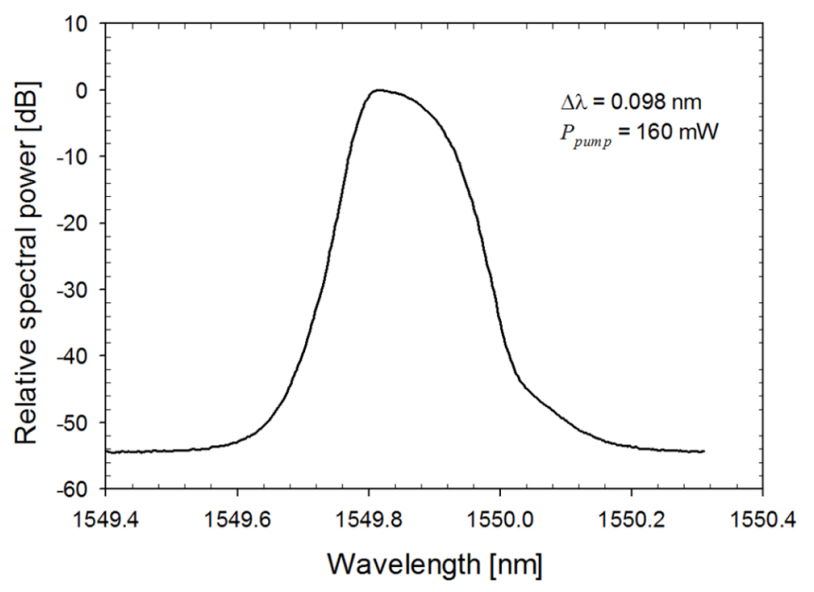

(a)

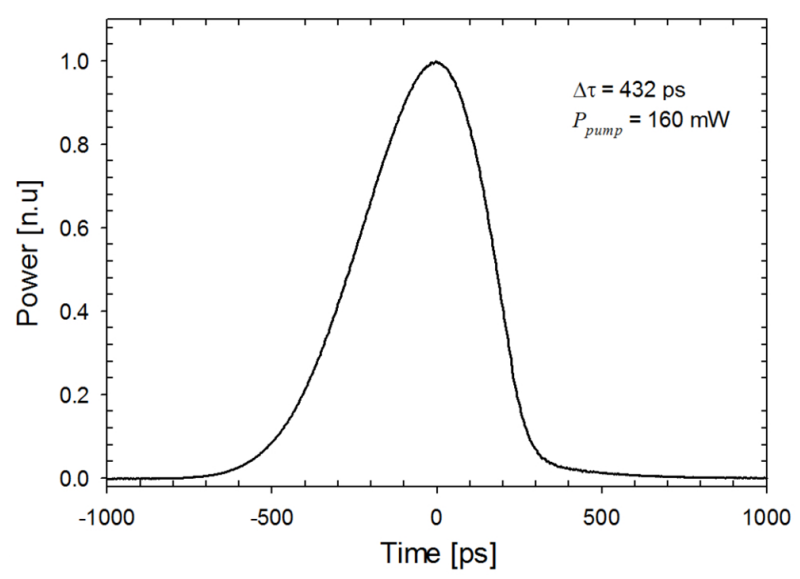

(b)

FIG. 3. (a) Optical spectrum and (b) time trace of the mode-locked pulses formed in the FSFL comprising a $110-\mathrm{MHz}$ downshifting AOM and an FBG BPF with $\Delta \lambda_{F}=$ $0.45 \mathrm{~nm}$. 


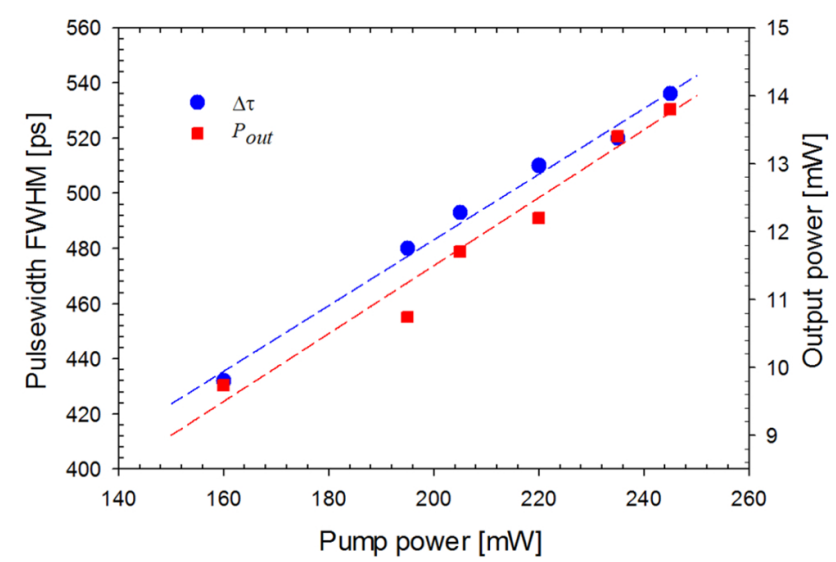

FIG. 4. Pulsewidth (FWHM) and output power as a function of the pump power. The dashed lines denote the linear fits of the measured data, respectively.

worth mentioning that once the laser was mode-locked, it was possible to keep the laser operating in the CPML regime while decreasing the pump power down to $\sim 160$ $\mathrm{mW}$. Below this value, mode-locking was no longer supported in the cavity. When the mode-locking was lost for the pump power of less than $\sim 160 \mathrm{~mW}$, the laser could not be mode-locked again until the pump power was set to in excess of $200 \mathrm{~mW}$. In fact, this pump-power hysteresis is a common feature observed in FSF mode-locked lasers [9, 17]. In addition, fundamental mode-locking was always achieved for any polarization states and pump powers, and no harmonic mode-locking was observed.

For comparison, Fig. 5 shows the pulse spectrum and time trace characteristics of the FSFL when a $200-\mathrm{MHz}$ downshifting AOM was incorporated into the cavity instead of the 110-MHz AOM. This shows significantly different lasing behaviors compared to those with the 110-MHz AOM. Modelocking was only obtained for the pump power of $\sim 250$ $\mathrm{mW}$ in this configuration. The significant increase of the self-starting threshold was due mainly to the $4-\mathrm{dB}$ of the excessive loss of the 200-MHz AOM in comparison with that of the $110-\mathrm{MHz} \mathrm{AOM}$. It is noteworthy that in this configuration there is a clear, half-separation between the consecutive pulses. The pulse spectrum formed a main peak with a spectral bandwidth of $0.04 \mathrm{~nm}$ and a broad shoulder on the longer wavelength side that is $\sim 10 \mathrm{~dB}$ below the level of the spectral maxima. We consider that this lasing regime is somewhat different from that observed in the previous case. We attribute the excessive loss of the $\mathrm{AOM}$, together with the increment in the frequency-shift quantity, to the reduced amplification of the spectral components located on the longer wavelength side of the spectrum. Thus, the shoulder formed on the longer-wavelength side remained $\sim 10-\mathrm{dB}$ lower than the spectral maxima. Consequently, it could not form a complete, consolidated bound state having a single-entity envelope as seen in Fig. 3(b).

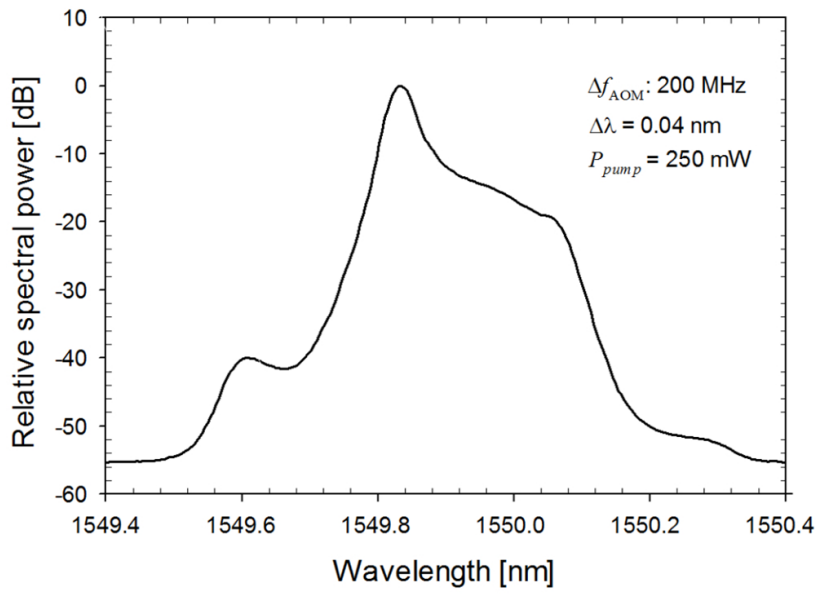

(a)

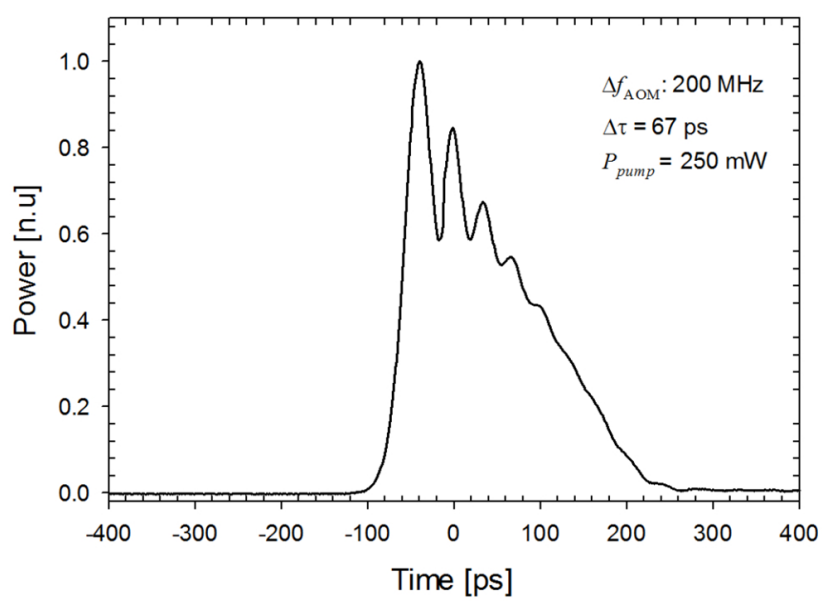

(b)

FIG. 5. (a) Optical spectrum and (b) time trace of the mode-locked pulses formed in the FSFL comprising a 200-MHz downshifting AOM and an FBG BPF with $\Delta \lambda_{F}=$ $0.45 \mathrm{~nm}$.

\section{CONCLUSION}

We have presented an all-fiberized, power-scalable, selfstarting sub-nanosecond mode-locked laser, which was based on a frequency-shifted-feedback ring cavity incorporating $2.3 \mathrm{~m}$ of an EDF, a $110-\mathrm{MHz}$ downshifting AOM, and a BPF of $0.45 \mathrm{~nm}$ (FWHM). Different from conventional MPML FSFLs, it could operate in the CPML regime and deliver an output power of 9 to $16 \mathrm{~mW}$ as proportional to pump power. The consolidated pulse with a single-entity envelope was formed thanks primarily to the inclusion of the BPF of 0.45-nm bandwidth into the cavity, which effectively hindered the formation of quasi-soliton pulses. Based on our experiment, the CPML regime was obtained when the bandwidth of BPF was 4 to 5 times broader than the spectral bandwidth of the laser signal. However, we 
are aware that this condition may change based on other parameters of the laser cavity, including the transfer function of the BPF, the frequency-dependent loss in the cavity, the dispersion of the cavity, the inversion level of the gain medium, etc. In the anomalous dispersion regime having negligible SPM effects, the continuously downshifted components undergo gradually elongated cavity round-trip times, so that they are successively accumulated in the trailing edge. This consequence, together with the balancing of gain and loss incurred by the EDF and BPF [13], leads to the asymmetries observed in the spectral and temporal shapes of the resultant pulse. The developed FSFL can have further power-scaling as well as pulse-energy scaling if higher pump is launched in the cavity. This can also be utilized as a masteroscillator for a power amplification system for achieving high-energy pulses.

\section{ACKNOWLEDGMENT}

The work was supported in part by the Ministry of Knowledge Economy (Project No.10040429).

\section{REFERENCES}

1. F. V. Kowalski, P. D. Hale, and S. J. Shattil, "Broadband continuous-wave laser," Opt. Lett. 13, 622-624 (1988).

2. D. J. Taylor, S. E. Harris, S. T. K. Nieh, and T. W. Hansch, "Electronic tuning of a dye laser using the acousto-optic filter," Appl. Phys. Lett. 19, 269-271 (1971).

3. O. G. Okhotnikov, "Multiwavelength picosecond frequencyshifted feedback laser with pulse control by a shaped-gain fiber amplifier," Opt. Lett. 23, 1459-1461 (1998).

4. A. Bellemare, M. Karásek, M. Rochette, S. LaRochelle, and M. Têtu, "Room temperature multifrequency erbium-doped fiber lasers anchored on the ITU frequency grid," J. Lightwave Technol. 18, 825-831 (2000).

5. J.-N. Maran, S. LaRochelle, and P. Besnard, "Erbium-doped fiber laser simultaneously mode locked on more than 24 wavelengths at room temperature," Opt. Lett. 28, 2082-2084 (2003).

6. H. Sabert and E. Brinkmeyer, "Pulse generation in fiber lasers with frequency shifted feedback," J. Lightwave Technol. 12, 1360-1368 (1994).

7. M. Romagnoli, S. Wabnitz, P. Franco, M. Midrio, L. Bossalini, and F. Fontana, "Role of dispersion in pulse emission from a sliding-frequency fiber laser," J. Opt. Soc. Am. B 12, 938-944 (1995).

8. F. Fontana, L. Bossalini, P. Franco, M. Midrio, M. Romagnoli, and S. Wabnitz, "Self-starting sliding-frequency fiber soliton laser," Electron. Lett. 30, 321-322 (1994).

9. M. Romagnoli, S. Wabnitz, P. Franco, M. Midrio, F. Fontana, and G. E. Town, "Tunable erbium-ytterbium fiber sliding-frequency soliton laser," J. Opt. Soc. Am. B 12, 72-76 (1995).

10. J. M. Sousa and O. G. Okhotnikov. "Short pulse generation and control in Er-doped frequency-shifted-feedback fibre lasers," Opt. Commun. 183, 227-241 (2000).

11. S. U. Alam and A. B. Grudinin, "Tunable picosecond frequency-shifted feedback fiber laser at $1550 \mathrm{~nm}$," IEEE Photon. Technol. Lett. 16, 2012-2014 (2004).

12. L. Lefort, A. Albert, V. Couderc, and A. Barthelemy, "Highly stable 68-fs pulse generation from a stretched-pulse $\mathrm{Yb}^{3+}$-doped fiber laser with frequency shifted feedback," IEEE Photon. Technol. Lett. 14, 1674-1676 (2002).

13. C. M. de Sterke and M. J. Steel, "Simple model for pulse formation in lasers with a frequency-shifting element and nonlinearity," Opt. Commun. 117, 469-474 (1995).

14. L. A. Vazquez-Zuniga and Y. Jeong, "Super-broadband noise-like pulse erbium-doped fiber ring laser with a highly nonlinear fiber for Raman gain enhancement," IEEE Photon. Technol. Lett. 24, 1549-1551 (2012).

15. L. E. Nelson, D. J. Jones, K. Tamura, H. A. Haus, and E. P. Ippen, "Ultrashort-pulse fiber ring lasers," Appl. Phys. B 65, 277-294 (1997).

16. L. F. Mollenauer, J. P. Gordon, and S. G. Evangelides, "The sliding-frequency guiding filter: an improved form of soliton jitter control,” Opt. Lett. 17, 1575-1577 (1992).

17. M. Nakazawa, E. Yoshida, and Y. Kimura, "Low threshold, 290-fs erbium-doped fiber laser with a nonlinear amplifying loop mirror pumped by InGaAsP laser-diodes," Appl. Phys. Lett. 59, 2073-2075 (1991). 